\title{
THE CAUCHY THEOREM FOR FUNCTIONS ON CLOSED SETS
}

\author{
PHILIP T. MAKER
}

The object of this paper is to extend the theorem of Cauchy to functions of a complex variable defined on any bounded closed set, $E$, by determining conditions on $f(z)$ in order that for certain coverings of $E$, $C_{n}$, and an extension of $f(z), f^{*}(z), \lim _{n \rightarrow \infty} \int_{c_{n}} f^{*}(z) d z=0$. It was suggested partly by the notion of a general monogenic function due to Trjitzinsky ${ }^{1}$ and partly by the measure theory methods of Menchoff ${ }^{2}$ and others, which succeed so well in lightening the restrictions on the real and imaginary parts of a complex function in order that $f(z)$ be regular.

Throughout this paper we shall consider only rectangles with sides parallel to the real and imaginary axes. A $C$-covering of a plane set $F$, denoted by $C$, will be a set of closed rectangles, possibly abutting, but nonoverlapping, which contain $F$. $c$ will denote the boundary of $C$. The covering $C_{n}$ is to be composed of rectangles $R_{m n}$ so that $C_{n}=\sum_{m} R_{m n}(m, n=1,2, \cdots)$.

1. The extension, $f^{*}(z)$. If $u(P)$ is a positive continuous function defined on the closed and bounded set $F$ in the plane, we shall let ${ }^{3}$ $u^{*}(P)=\max _{Q \in F} u(Q)\{2-d(P, Q) / d(P, F)\}$ for $P$ not in $F$, and $u^{*}(P)=u(P)$ for $P$ in $F$, where $d(P, Q)$ denotes the distance from $P$ to $Q$ and $d(P, F)$ the distance from the set $F$ to $P$. In general, if $u(P)$ is continuous, since $u(P)=(u(P)+|u(P)|) / 2-(|u(P)|-u(P)) / 2$, that is, since $u(P)$ is the difference of two continuous positive functions, $u^{*}(P)$ will denote the extension of $u(P)$ obtained by extending as before these parts. If $f(z)(=u(x, y)+i v(x, y))$ is defined on a bounded closed set and continuous, $f^{*}(z)$ will denote $u^{*}(x, y)+i v^{*}(x, y)$.

Lemma 1. If $u(P)$ is defined on a bounded closed set $F$ and $\mid u(Q)$ $-u(P) \mid<M(P) d(P, Q)$ where $M(P)$ is a finite function of $P$ defined on $F$, then $\left|u^{*}(P)-u^{*}(Q)\right|<20 M(P) d(P, Q)$, for $P$ in $F$ and $Q$ arbitrary.

Presented to the Society, December 29, 1941; received by the editors February $25,1942$.

${ }^{1}$ W. J. Trjitzinsky, Théorie des Fonctions d'une Variable Complexe Définies sur des Ensembles Généraux, Annales Scientifique de L'École Normale Supérieure, Paris, 1938, p. 120.

${ }^{2}$ D. Menchoff, Les Conditions de Monogénéité, Actualités Scientifiques et Industrielles, no. 329, Paris, 1936.

${ }^{3}$ S. Bochner, Fourier Lectures, 1936-1937, Princeton, p. 62. 
Proof. Consider first the case for which $u(P) \geqq 0$. If $Q$ is any point not in $D$, if $Q_{0}$ is a point in $F$ for which $d\left(Q, Q_{0}\right)=d(Q, F)$, and if, of the points $S$ satisfying the inequality $d(S, Q) \leqq 2 d\left(Q, Q_{0}\right), R$ is the point where the maximum of $u$ is attained, then $u^{*}\left(Q_{0}\right) \leqq u^{*}(Q)$ $\leqq u^{*}(R)$. Hence

$$
\begin{aligned}
\left|u^{*}(Q)-u^{*}(P)\right| & \leqq\left|u^{*}(Q)-u^{*}(R)\right|+\left|u^{*}(R)-u^{*}(P)\right| \\
& \leqq\left|u^{*}\left(Q_{0}\right)-u^{*}(P)\right|+2\left|u^{*}(R)-u^{*}(P)\right| \\
& <M(P) d\left(Q_{0}, P\right)+2 M(P) d(R, P) .
\end{aligned}
$$

It is easily verified that $d\left(Q_{0}, P\right) \leqq 2 d(Q, P)$ and $d(R, P) \leqq 4 d(Q, P)$, so that $\left|u^{*}(Q)-u^{*}(P)\right|<10 M(P) d(P, Q)$ and the lemma is proved for case of $u(P)$ positive. In the general case, $u(P)=(|u|+u) / 2$ $-(|u|-u) / 2=g(P)-h(P)$, where $g$ and $h$ are positive functions, and satisfy the conditions of the lemma, so that for $P$ in $F$ and $Q$ arbitrary $\left|g^{*}(Q)-g^{*}(P)\right|$ and $\left|h^{*}(Q)-h^{*}(P)\right|$ are each less than $10 M(P) d(P, Q)$. Hence for $u^{*}=g^{*}-h^{*}$, it readily follows that $\left|u^{*}(Q)-u^{*}(P)\right|<20 M(P) d(P, Q)$, and the proof of the lemma is complete.

2. Bounded derivatives. We shall use the following fundamental lemma: ${ }^{4}$

Lemma 2. Let $w(x, y)$ be a real, continuous function defined in the square $S$, the sides of which are parallel to the coordinate axes, and let $F$ be a closed set in $S$ and such that

$$
\begin{aligned}
& |w(x+h, y)-w(x, y)| \leqq M|h|, \\
& |w(x, y+k)-w(x, y)| \leqq M|k|
\end{aligned}
$$

for all points $(x, y)$ in $F$ and for all points $(x+h, y),(x, y+k)$ of the square $S$, where $M$ is a constant. Finally let $R$ be the least rectangle with sides parallel to the axes containing $F^{5}$

Under these conditions ${ }^{6}$ the following inequalities hold:

$$
\begin{aligned}
& \left|\int_{x_{1}}^{x_{2}}\left[w\left(x, y_{2}\right)-w\left(x, y_{1}\right)\right] d x-\iint_{F} \frac{\partial w}{\partial y} d x d y\right| \leqq 5 M m(S-F), \\
& \left|\int_{y_{1}}^{y_{2}}\left[w\left(x_{2}, y\right)-w\left(x_{1}, y\right)\right] d y-\iint_{F} \frac{\partial w}{\partial x} d x d y\right| \leqq 5 M m(S-F)
\end{aligned}
$$

where $\left(x_{1}, y_{1}\right),\left(x_{2}, y_{1}\right),\left(x_{2}, y_{2}\right)$ and $\left(x_{1}, y_{2}\right),\left(x_{1} \leqq x_{2}, y_{1} \leqq y_{2}\right)$ are the

${ }^{4}$ For the proof of this lemma, cf. loc. cit., p. 10.

5 The "least rectangle" may be only a segment or a point.

${ }^{6}$ The conditions on $w$ imply the existence of the partial derivatives a.e. in $F$. 
corners of the rectangle $R$ and $m(S-F)$ is the measure of the set $S-F$, which is composed of points of $S$ not in $F$.

THEOREM 1. Let $f(z)(=u(x, y)+i v(x, y))$ be defined on the bounded closed set $E$, and let $R$ be a rectangle with sides parallel to the axes containing at least one point of $E$ on each side. If (letting $F=E \cdot R$ )

(1) for all $z$ and $z+h$ in $F$, and a constant $B$,

$$
\left|\frac{f(z+h)-f(z)}{h}\right|<B
$$

(2) the Cauchy-Riemann equations hold a.e. (almost everywhere) in $F$, where the partial derivatives of $u$ and $v$ exist, ${ }^{7}$ then $\left|\int_{r} f^{*}(z) d z\right|$ $<400 B m(S-F)$ where $r$ is the boundary of $R$, and $S$ is a square of least area containing $R$.

Proof. If $h=k+i l$, condition (1) implies

$$
\left|\frac{u(x+k, y+l)-u(x, y)}{h}\right|<B,
$$

and a similar condition on $v(x, y)$, for every point $z$, and $z+h$, in $F$. According to Lemma 1,

$$
\left|\frac{u^{*}(x+k, y+l)-u^{*}(x, y)}{h}\right|<20 B,
$$

for each point $z$ in $F$, and $z+h$ in $R$. Hence by Lemma 2,

$$
\left|\int_{x_{1}}^{x_{2}}\left[u^{*}\left(x, y_{2}\right)-u^{*}\left(x, y_{1}\right)\right] d x-\iint_{F} \frac{\partial u^{*}}{\partial y} d x d y\right|<100 B m(S-F),
$$

$\left(x_{1}, y_{1}\right)$ and $\left(x_{2}, y_{2}\right)$ being corners of $R$. Similar inequalities for $u^{*}(x, y)$ with respect to $y$, and $v^{*}(x, y)$ with respect to $x$ and $y$ also hold. But

and

$$
\int f^{*}(z) d z=\int_{r} u^{*} d x-v^{*} d y+i \int_{r} v^{*} d x+u^{*} d y
$$

$$
\int_{r} u^{*} d x=-\int_{x_{1}}^{x_{2}}\left[u^{*}\left(x, y_{2}\right)-u^{*}\left(x, y_{1}\right)\right] d x \text {. }
$$

7 This is in no way a further restriction on $E$, for almost all points of any measurable plane set are points of linear density for it both in the direction of the $x$-axis and in that of the $y$-axis.

The condition that the limits, $\lim _{h \rightarrow 0}(f(z+h)-f(z)) / h$, as $z+h$ approaches $z$ through points of $E$ along either of two curves having non-collinear tangents at $z$, should be equal, is equivalent to the condition (2) in the presence of (1). For (1) and (2) imply that $f^{*}(z)$ is monogenic a.e. in $E$ (Menchoff, loc. cit., Theorem 2, p. 27 and Theorem 5, p. 23). 
Let $\epsilon^{\prime}=\int_{x_{1}}^{x_{2}}\left[u^{*}\left(x, y_{2}\right)-u^{*}\left(x, y_{1}\right)\right] d x-\iint_{F}\left(\partial u^{*} / \partial y\right) d y d x$. Then $\int_{r} u^{*} d x$ $=-\iint_{F}\left(\partial u^{*} / \partial y\right) d y d x-\epsilon^{\prime}$. Taking into account similar reasoning for the other parts of $\int_{r} f^{*}(z) d z$ we have

$$
\begin{aligned}
\int_{r} f^{*}(z) d z= & -\iint_{F}\left(\frac{\partial u^{*}}{\partial y}+\frac{\partial v^{*}}{\partial x}\right) d x d y \\
& +i \iint_{F}\left(\frac{\partial u^{*}}{\partial x}-\frac{\partial v^{*}}{\partial y}\right) d x d y+\epsilon
\end{aligned}
$$

where $|\epsilon|<400 B m(S-F)$. Since the Cauchy-Riemann equations hold a.e. in $F, \int_{r} f^{*}(z) d z=\epsilon$, and the proof of the theorem is complete.

Corollary. Let $f(z)$ be defined on the bounded closed set $E$ with a bounded derivative there. Let $S=\sum_{m} S_{m}$ be a $C$-covering of $E$ by squares with $R_{m}$ the least rectangle within $S_{m}$ containing $S_{m} \cdot E$. Then there is a constant $B$ for which $\sum_{m}\left|\int_{r_{m}} f^{*}(z) d z\right|<400 B m(S-E)$, and if $S \rightarrow E$, $C=\sum_{m} R_{m} \rightarrow E$, and $\lim _{C \rightarrow E} \int_{c} f^{*}(z) d z=0$.

3. Derivatives finite, except for a denumerable set. We prove this theorem:

Theorem 2. If $f(z)$ is defined and continuous on the bounded closed set $E$, and if, except for a denumerable number of points, $\lim \sup _{h \rightarrow 0}|(f(z+h)-f(z)) / h|<\infty$, and the Cauchy-Riemann equations hold a.e. where the partial derivatives of $u$ and $v$ exist, then there is a sequence of C-coverings, $\left\{C_{n}\right\}$, for which $\lim _{n \rightarrow \infty} \sum_{m}\left|\int_{r_{m n}} *^{*}(z) d z\right|=0$.

Proof. Define $I(C)=\sum_{m}\left|\int_{r_{m}} f^{*}(z) d z\right|$. If for every point $z$ of $E$ there is a neighborhood $N(z)$ such that for every closed subset of $E$ in $N$, there is a sequence of coverings $\left\{C_{n}\right\}$ for which $\lim _{n \rightarrow \infty} I\left(C_{n}\right)=0$, then by the Heine-Borel theorem there exists a sequence of coverings of $E$ with the property mentioned in the theorem. The proof will be complete therefore, if we show that there is such a neighborhood for each point of $E$. Let $P$ be those points of $E$ such that in every neighborhood of $z$ there is a closed subset of $E$ for which there is no sequence of $C$-coverings, $\left\{C_{n}\right\}$, for which $\lim _{n \rightarrow \infty} I\left(C_{n}\right)=0$. We shall assume that $P$ is not empty and show that this leads to an absurdity.

Let $P_{m}(m=1,2, \cdots)$ be the points of $P$ for which each of the absolute values,

$$
\begin{array}{ll}
\left|u^{*}(x+k, y)-u^{*}(x, y)\right|, & \left|v^{*}(x+k, y)-v^{*}(x, y)\right|, \\
\left|u^{*}(x, y+k)-u^{*}(x, y)\right|, & \left|v^{*}(x, y+k)-v^{*}(x, y)\right|
\end{array}
$$

is less than or equal to $m|k|$ for $|k| \leqq 1 / m, k$ a real number. Since 
$u^{*}$ and $v^{*}$ are continuous and $P$ is closed, $P_{m}$ is closed. Since at each point of $E$, except for a denumerable set $H$, the partial derivates are finite, $P=\sum_{m} P_{m}+P \cdot H$. By Baire's ${ }^{8}$ theorem, there is an isolated point of $P$ in $H$, or for some integer $N$ there is a point $z_{0}$ in $P$, the center of a square $S$ which contains only points of $P$ which are in $P_{N}$. The former alternative is quickly dismissed as impossible; we proceed on the basis of the latter, and let $F$ be any closed subset of $E \cdot S$. Subdivide the sides of $S$ into $n$ equal parts, $n>2 N$, and obtain the squares $S_{j}\left(j=1,2, \cdots, n^{2}\right) . \epsilon$ being given, choose $n$ so great that the squares $\bar{S}_{j}$ which contain points of $F \cdot P$ satisfy the inequality, $m\left(\sum_{j} \bar{S}_{j}-P \cdot S\right)<\epsilon / 800 N$. If $\bar{R}_{j}$ is the least rectangle containing $P \cdot \bar{S}_{j}$, and $\bar{C}$ is the covering $\sum_{j} \bar{R}_{j}$, by Theorem $1, I(\bar{C})$ $<400 N \sum_{j} m\left(\bar{S}_{j}-P \cdot \bar{S}_{j}\right)<\epsilon / 2$. Since $I(R)$ is a continuous function of $r, \bar{C}$ may be extended by the addition of more small rectangles, so that, if $C^{\prime}$ is the new covering, $I\left(C^{\prime}\right)$ remains less than $\epsilon / 2$, but so that the points of $F \cdot P$ are inner points of the covering. The part of $F$ not already covered (denote it by $G$ ) is such that its closure contains only points $z$ of $F$ for which there is some neighborhood $N(z)$ with the property that every closed subset of $F$ in $N$ can be $C$-covered, say by $C_{n}(z)(n=1,2, \cdots)$ and $\lim _{n \rightarrow \infty} I\left(C_{n}(z)\right)=0$. Let $S(z)$ be a square with $z$ as center entirely within $N(z)$. Of these squares a finite number, $k$, cover $G$, and within each of these is a covering, $C(z)$, of $G$ for which $I(C(z))<\epsilon / 2 k$. Hence $G$ is $C$-covered by a covering $C$ for which $I(C)<\epsilon / 2 . \quad F$ is therefore $C$-covered by $C+C^{\prime}$ for which $I\left(C+C^{\prime}\right)<\epsilon$, so that $z_{0}$ cannot belong to $P$, contrary to assumption. This completes the proof of Theorem 2.

Corollary. If $f(z)$, defined on the bounded closed set $E$ and continuuous there, has a derivative at each point except at most a denumerable set, there is a sequence of $C$-coverings of $E$ with $E$ as their limit for which $\lim _{n \rightarrow \infty} \int_{C_{n}} f^{*}(z) d z=0$.

DUke University

${ }^{8}$ S. Saks, The Theory of the Iniegral, New York, 1937, p. 54. 